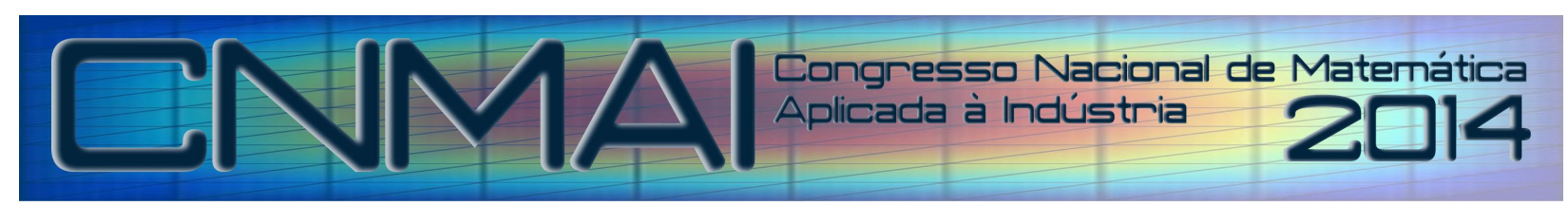

18 a 21 de novembro de 2014, Caldas Novas - Goiás

\title{
SYSTEM FOR MAPPING AN ESTIMATE ZONE OF ACTUATION OF ULTRASONIC SENSORS
}

\author{
Rômulo Muriel Mesquita de Oliveira, romulooliveira@gmail.com¹ \\ Marcelo Henrique Stoppa, mhstoppa@pq.cnpq.br ${ }^{2}$ \\ Núbia dos Santos Saad, nubia@ufu.br ${ }^{3}$
}

\footnotetext{
${ }^{1}$ Universidade Federal de Goiás, Av. Dr. Lamartine Pinto de Avelar, 1120, Setor Universitário, Catalão-GO

${ }^{2}$ Universidade Federal de Goiás, Av. Dr. Lamartine Pinto de Avelar, 1120, Setor Universitário, Catalão-GO

${ }^{3}$ Universidade Federal de Uberlândia, Av. João Naves de Ávila, 2121, Santa Mônica, Uberlândia-MG
}

\begin{abstract}
It is possible to see a considerable growth in the application of sensors in processes and products, seeking for automation, quality gain or to increase safety. Among the vast array of sensors, one of the most used is the ultrasonic sensor. It works based on a measurement of the time spent by a sound wave that travels from a transmitter transducer, reflects on the surface of an object and returns to a detector, commonly used for determining distance. Despite being a well-known principle, it is not trivial to determine the total coverage space of the ultrasonic sensor. Companies that produce ultrasonic sensors, generally, specify the actuation distance or the range of the sensor in its datasheet. However, the information about the working space of the sensors is not presented, only a linear distance or a planar region of operation is shown. Considering projects where the $3 D$ zone of actuation of the sensor is essential, this paper presents a system capable of measuring this zone for ultrasonic sensors, thus ensuring greater reliability in their application in situations that requires security. To define the region where the ultrasonic waves can detect an object, a system was developed, allowing the mapping of the three-dimensional zone. The system consists of a metal structure, on which a robot, built with LEGOC's kit, positioning an object that can be captured by the ultrasonic sensor. The object moves in three mutually perpendicular axes, making a right prism with a rectangular base $(1 \mathrm{~m} x 1 \mathrm{~m}$ $x 4 m$ ). The ultrasonic sensor is positioned in the center of the first plane and captures the object moving in the structure space. After the mapping it was possible to generate a graphic with the estimate zone of activity of the sensor, which helped to define the beam of the sensor, contributing in the identification of the opening angle and maximum range of detection. This method can be also performed for evaluation of sensors applied in vehicular safety systems, identifying the safe zone of operation.
\end{abstract}

Keywords: ultrasonic sensor, actuation zone, mapping, security system

\section{INTRODUCTION}

Sensors are responsible to collect variables in the environment and provide the results as electrical signals. (Mágori, 1994).

The term ultrasound is used to define acoustic waves with frequency audible higher than the human capacity, i.e. above 20,000 cycles per second (Webster, 1999). Also known as ultrasonic signals, the inaudible sound waves are captured through a transducer, which, due to resonance, convert acoustic energy into electrical and vice versa (Thomazini, 2005).

Stimulated by the high application potential, and low cost, ultrasonic sensors are devices that, through electrical transducers, make use of the pulse-echo method for measuring distance (Mágori, 1994). This method is able to detect the distance of an object in function of time between emission and receipt of the reflected wave (Bastos, 1999). To determine the distance, as shown in Eq. (1), the sensor measures the transit time required for an ultrasonic wave travels the path roundtrip. Once this time interval is obtained, through the reflected-echo into the receiving transducer, the time is converted into distance.

$$
d=\frac{c t}{2}
$$


In eq. (1), the term $d$ refers to the distance from the sensor to the object, $c$ the speed of sound in air ant $t$ the time of the ultrasonic pulse, considering roundtrip path. The last two variables are divided by two, in function of the roundtrip route that the acoustic wave takes, resulting in the distance between object and sensor (Rastogi and Mehra, 2012). In industry, ultrasonic sensors are commonly applied, providing among other benefits, control of operations, measurements and detections (Mágori, 1994). Ultrasonic proximity sensors, in turn, are used to verify levels fluids pass, count products in an assembly line and detecting the presence of people. However, for applications that requires a higher level of reliability there is no sufficient information for the employment of the sensor. Among other difficulties, highlights the limitations of knowing the acoustic space where the sensor works and its maximum range. In additional, to use an ultrasonic sensor in a wrong position can compromise its application. In some cases, to reduce positional uncertainty, more ultrasonic sensors are applied (Kim, 2010).

Evaluation of the reliability of the proximity measurements made with ultrasonic systems is, in general, difficult (Majchrzak, 1999). Although, the absence of information could harm in obtaining desired results.

Thus, using ultrasonic sensors to meet specific applications, uncertainties may arise regarding the definition of range, opening angle and actuation zone. Ultrasonic sensor has non-linear characteristics, so the information about the detection zone of the sensor and how the opening angle is positioned (horizontal or vertical) are essential for proper application (Mohammad, 2009).

The technical information provide by manufacturers ultrasonic sensors, generally presents a flat surface (as a top view), which shows the limits of detection, although the information do not guarantee the correct functioning of the sensor. The actuation zone cannot be represented as a top view, because the shape of this zone is not flat. The drawbacks are not related to the product, but are inherent to the principle of ultrasonic range finders (Borenstein, 1988). Furthermore, the distance of action mentioned by the suppliers, in some cases, are not effective and the distance measurements has a blind area, that provide inaccuracies in applications that require safety (Zang et.al. 2004).

Another application that requires better specification of the ultrasonic sensor actuation zone is the active vehicular safety, which includes sensors in vehicles to minimize the possibility of collision. The result of this paper contributes with important data for this kind of application, not limited only to it. The benefits would aid in choosing the best sensor to be used and possibility to assess the actuation zone, contributing to the correct positioning in the vehicle.

Therefore, this article aims to identify the beam of ultrasonic sensors, through the development of a system able to detect the actuation zone.

\section{DEVELOPMENT}

Due to the difficulty in identifying the actuation zone of ultrasonic sensors, a method was developed for mapping an estimate zone of actuation, ensuring accuracy in sensing applications. Normally, in the technical specification of the sensors is possible to find, among other information, the electrical operating parameters, connections and beam pattern. For this last, apart for mentioning the detection distance (mininum and maximum range), the technical specifications of the suppliers usually brings a chart containing the horizontal area of operation of the sensors, as shown in figure 1 .

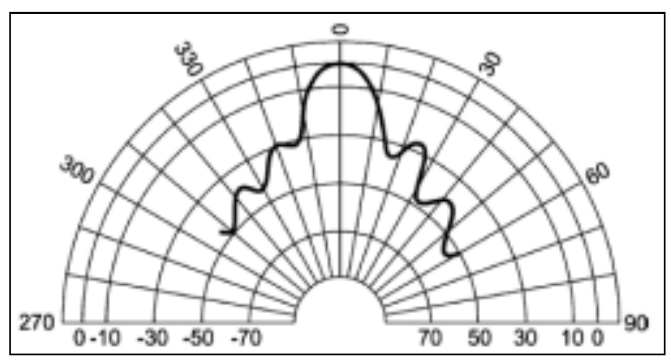

Figure 1. Typical characteristic of a horizontal section of the measuring beam for a 600-series Polaroid sensor (Majchrzak et al., 2009)

In this context, is possible to see in Figure 1, that the horizontal actuation zone (top view) of the ultrasonic sensor 600-series Polaroid, has an opening angle of approximately 120 degrees, however, if this zone also behaves similarly vertically, forming a cone whose vertex coincides with the point where the sensor is positioned, it is not evident.

There is difference between the actuation zone, defined by the supplier and the effective actuation zone, which can be referenced respectively as, nominal sensing distance and operational sensing distance. Nominal sensing distance (Sn) is the maximum distance that the sensor can operate, however despises weather forecast and voltage variations caused by processes inherent in its manufacture. Sensor operating distance $(\mathrm{Sa})$ is the distance that can be operated safely, considering the weather forecast, voltages and variations in industrialization (Fuentes, 2005). Commonly in specifications of ultrasonic sensors is highlighted the nominal sensing area, which does not guarantee the correct functioning of the sensor, with the possibility of losses on projects that require detection reliability.

For this study, two sensors were considered, one produced by Elec Freaks, named HC-SR04 and another marketed by LEGO Mindstorms, Model 9846, according to the figure 2. 


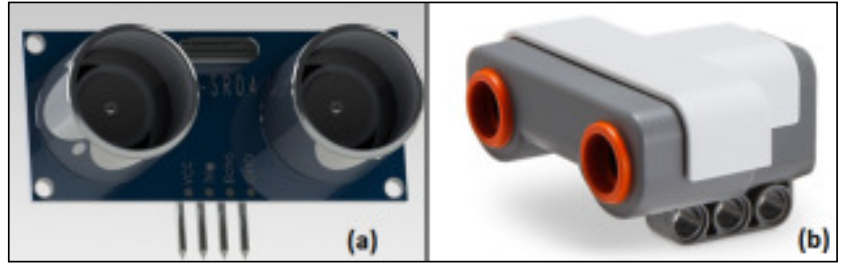

Figure 2. Ultrasonic Sensor: (a) HC-SR04; (b) 9846

According to the technical specifications (datasheet) of Elec Freaks, the HC-SR04 sensor provides a range of actuation between 20 and $4000 \mathrm{~mm}$, operating with an opening angle of 15 degrees, while the LEGO's sensor, according to the specifications found at the website, has a maximum range of $2500 \mathrm{~mm}$. The horizontal area of action of both sensors used in this study is not provided by the suppliers, however, for comparison purposes, the mapping was carried out under these conditions, in a way that the plane that shows the results of actuation limits, can be used to compare with the 3D actuation zone.

To obtain the detection area, for both sensors, was used the method of displaces a square metal plate of $40 \mathrm{~mm}$, in front of the sensor, evaluating the horizontal plane, in order to identify their performance limits, as shown in figure 3 , where a top view of the area of operation is presented.

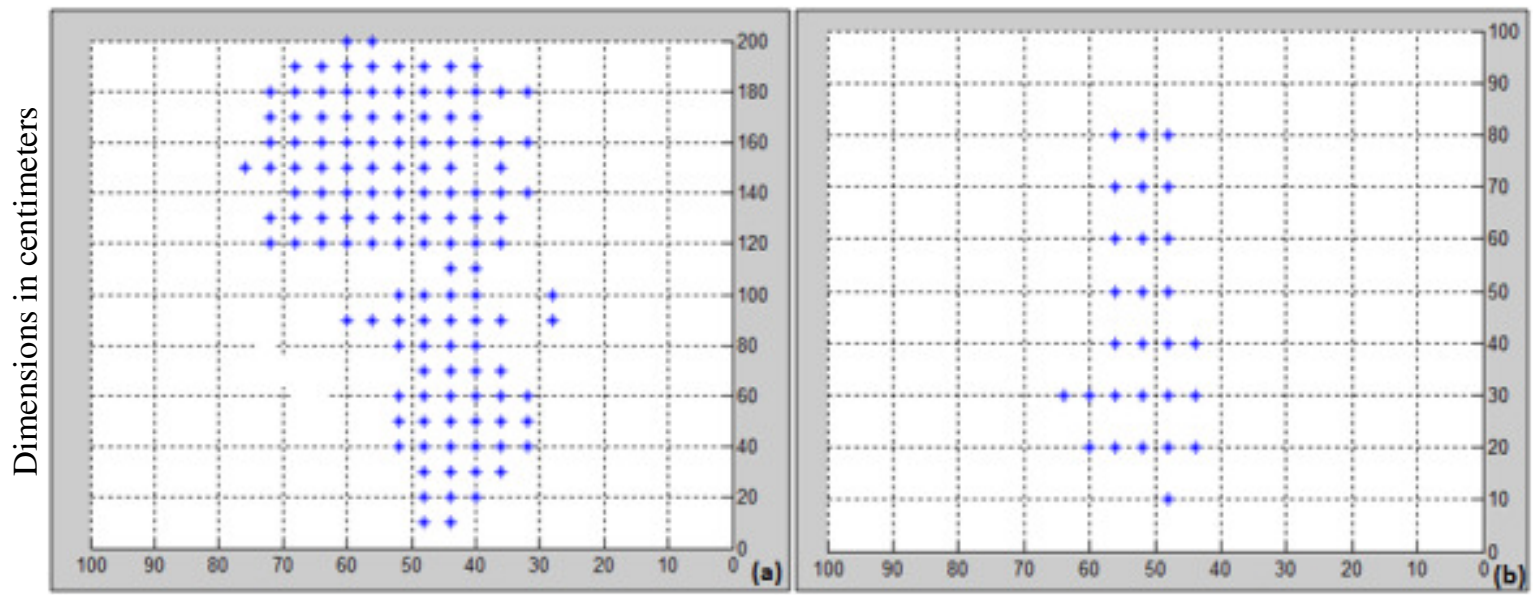

Figure 3. Top view of the actuation zone for HC-SR04 sensor (a) and 9846 LEGO's sensor (b)

The horizontal mapping of the zone of operation of the sensors adopted in this article, as figure 3 , transmit a notion of the range of the sensors and their detection area, however, still remains unclear if the sensors are able to identify objects above or below this area. Only the $3 \mathrm{D}$ actuation zone of the sensor could confirm its performance in the detection of objects.

\subsection{Proposal method}

The proposal to scan and identify the actuation zone of the ultrasonic sensor is based on an experimental method, where an object is moved in front of the sensor. Through the displacements, when any distance is captured, the results and position are stored, generating a matrix with the sensing results. The final matrices are composed by only two results, zero (0) when there is no detection and one (1) when the object entering in the detection zone.

Therefore, to identify the possible detection zone, an orthogonal cartesian structure was developed, capable of ensuring the displacement of the object without interfering in the detection of the sensor. This structure was built of metal bars of rectangular cross section $20 \times 30 \mathrm{~mm}$, with steel sheet of $0.7 \mathrm{~mm}$ thickness. The structure was designed with dimensions of 1000x1000x4000 mm (height, width and length), as shown in figure 4. 


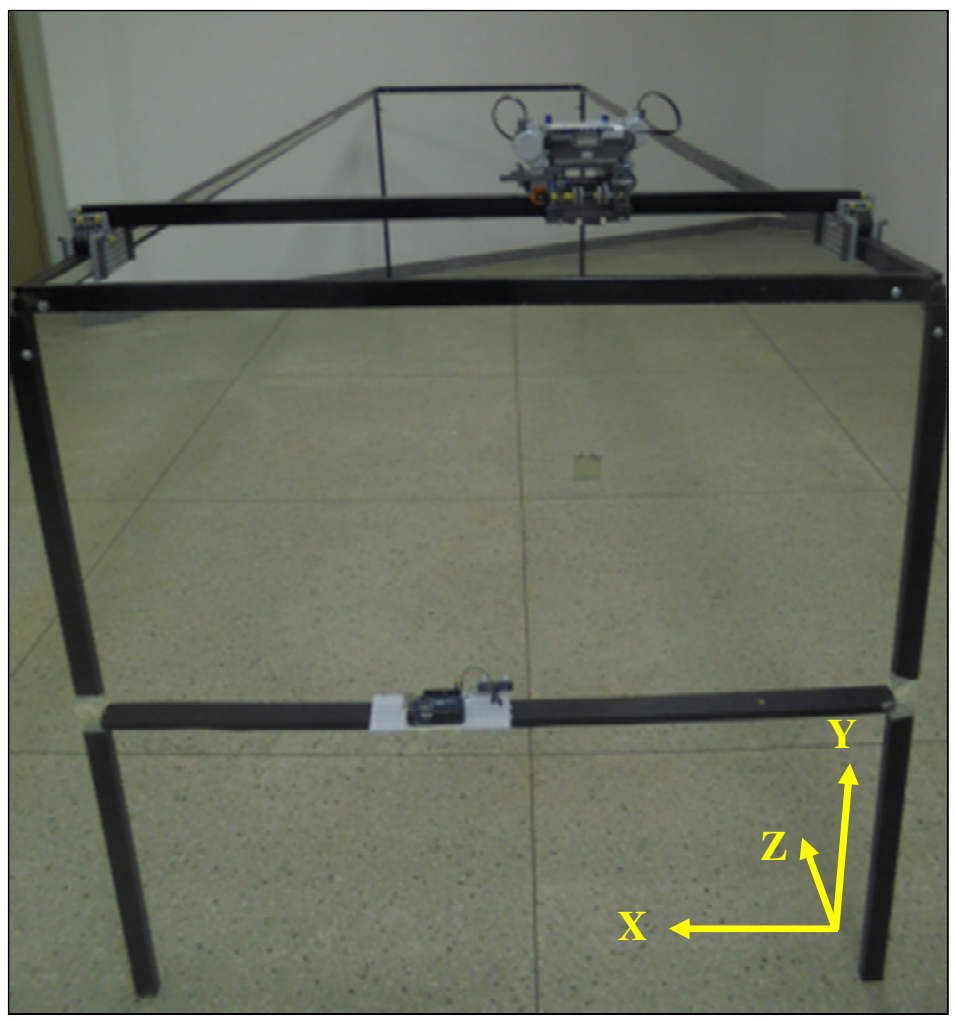

Figure 4. Isometric view of the structure with the respective axes

Above the structure, moves a bar, made with the same material of the structure, which moves in the $\mathrm{Z}$ axis, through pulleys with manual override. A LEGO robot was positioned over the bar, according to Figure 5, which is responsible for moving the object in the axes $\mathrm{X}$ and $\mathrm{Y}$, enabling the scanning process within the structure space.

This robot moves a square metal plate through the $\mathrm{X}$ and $\mathrm{Y}$ axes, which has the dimensions of $40 \mathrm{~mm}$ and a thickness of $4.3 \mathrm{~mm}$, making a scan on each plane $\mathrm{XY}$, perpendicular to the $\mathrm{Z}$ axis. The robot moves the plate in 625 points, for each cross section of the prismatic rectangular structure. The displacement occurs moving the plate 25 times in vertical and 25 times in horizontal. Two motors were installed on the robot, one carries out its own movement on the bar ( $\mathrm{X}$ axis) and the other is responsible for moving the plate ( $\mathrm{Y}$ axis). The metal plate was fixed on a spool that collects or releases the plate into the structure, to be detected by the sensor. A nylon yarn was used with $0.25 \mathrm{~mm}$ thick, which does not interfere with detection of the ultrasonic sensor.

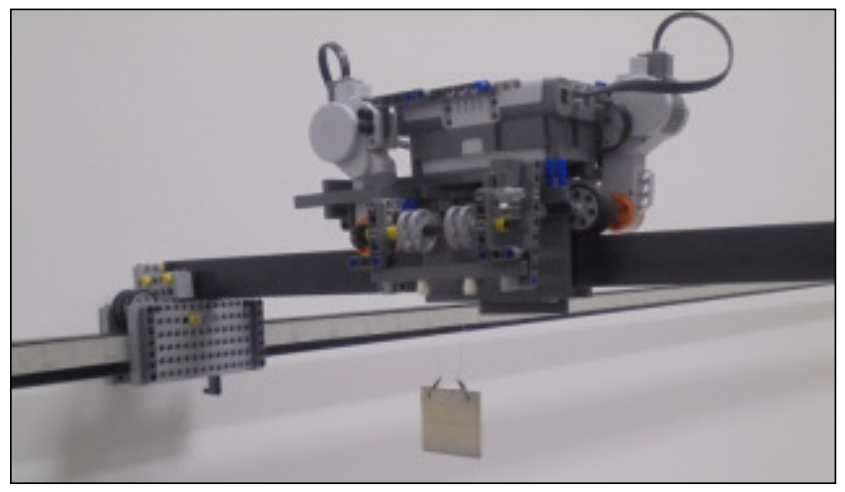

Figure 5. LEGO Robot moving the plate into the structure

Relative to the sensor, it is positioned on the center of the first plane (XY) of the structure, which contains a horizontal bar for accommodation sensors, as shown in figure 4, where they remain pointed towards the inside of the structure. The sensors used in this experiment do not present in their datasheets, actuation limits greater than the space provided by the structure, not compromising the tests. Moreover, been the sensor in actuation, positioned on the bar of accommodation, aiming for the center of the structure, no distance was detected, which proves there is no interference between the structure and the actuation zone.

The ultrasonic sensors, HC-SR04 and 9846, present in its datasheet an actuation limit lower than the space available in the structure, so they were used to validate the method. These sensors, as shown in Figure 2, are similar, containing 
two transducers, one transmitter and one receiver, both operates at a frequency of $40 \mathrm{KHz}$. However, in function of the connections that each one adopts, different methods for mapping were utilized. For the HC-SR04 sensor was used an Arduino, micro controller that manages the sensor readings while the robot moves the object. For the 9846 sensor was used a connection with the Brick NXT, LEGO's controller that manages communication with the robot and sends results to the software.

For the first experiment, for mapping the 9846 sensor actuation zone, were used two Bricks, one acting as a slave, which moves the object, and the other as master, which reads the position of the object at each point of the 3D mesh. The communication between the Bricks was established by Bluetooth technology. During the execution of tests, the sensor 9846 remains connected to the masters, which is responsible for requesting the sensor reading and sending commands to the slave for displacements.

After the Bluetooth connection be established between the Bricks, the program determines that the ultrasonic sensor, connected to the master, makes the first scan of the object, then immediately sends a command to the slave controller, for moving vertical or horizontally. Thus, for 24 times the slave receives commands for moving the plate vertically, providing the sensor reading. Then the master sends the command to the slave to retract the plate, so that the slave moves to the second column, starting a new scan. In this way, the slave robot moves the plate across the mesh structure, passing through the points that will be evaluated by the sensor. After each movement of the plate, the slave returns with another command to the master, informing that the displacement was performed and the sensor reading can be executed.

For capture the results the functionality Datalogging of LEGO Mindstorms system was used, which allows to extract data from experiments. i.e., between each movement and stop of the plate, the sensor reads the distance, which is stored by the datalogging. Once predefined the coordinates to be covered by the plate, it is necessary just to compare the results obtained in the sensor reading, with the positions of the 3D mesh.

The software used to building the displacement program of the robot, the reading sensor and storage results program, was the LEGO Mindstorms NXT 2.0, student version. The program was designed to move the plate in all predetermined points of the $\mathrm{X}$ and $\mathrm{Y}$ axes, constructing a grid divided into $40 \mathrm{~mm}$ for each plane.

The second experiment, for the HC-SR04 sensor, consists of use the same robot for displacement of the plate, synchronized with the sensor reading time. For this case, the sensor is connected to an Arduino micro controller. The robot is not paired with the micro controller, the execution of read and displacement occurs between both synchronously, where after the start of both systems, the Arduino will request a sensor reading, wait for 3 seconds and repeat the scanning. This time is sufficient to the robot shift the position of the object. The flow of movement of the object is similar to the first experiment, where the robot releases the object vertically, collecting at the end of the twenty-fifth position and moving to the next column to be mapped. The results are stored by the Serial Monitor of the Arduino, which are subsequently ordered according to pre-defined coordinates.

The free software used for build the program, responsible for the sensor reading was the Arduino 1.0.5-r2. To generate the graphs, which show the points where the object was detected, were used the Matlab Software, version 7.8.0.347 (R2009a).

For both experiments, the tests were applied with the plate perpendicular to the sensor. It was not considered the possibility of detecting the plate in different angles. The test was conducted in an enclosed area where the environmental conditions remained constant with temperature around 25 degrees Celsius, free from wind, noise and any other factor that could influence the results.

\section{RESULTS}

Two sensors were used for this experiment, the model 9846, from LEGO and the model HC-SR04 from Freaks Elec. Both have different specifications, however are commonly employed in academic environments, such as robotic projects, or those that require some kind of measurement and control.

\subsection{First experiment}

According to the specifications of LEGO, the ultrasonic sensor, model 9846, is capable to measure distance of objects up to $2,500 \mathrm{~mm}$. The scan was performed in a space of $1000 \mathrm{~mm}^{3}$, using the steel plate of $40 \mathrm{~mm}^{2}$. For the $\mathrm{Z}$ axis of the structure, in this experiment, it was divided in many planes, considering a distance of $100 \mathrm{~mm}$ from one to another. This planes (X,Y), are paralleled with the sensor plane and contain all the X and Y positions of the coordinate. In this first experiment, were analyzed ten planes. In each one of these, the plate were moved, creating a mesh of points in $\mathrm{X}$ and $\mathrm{Y}$, forming through the detection points, an estimate zone of actuation, which result can be seen in figure 6 . 


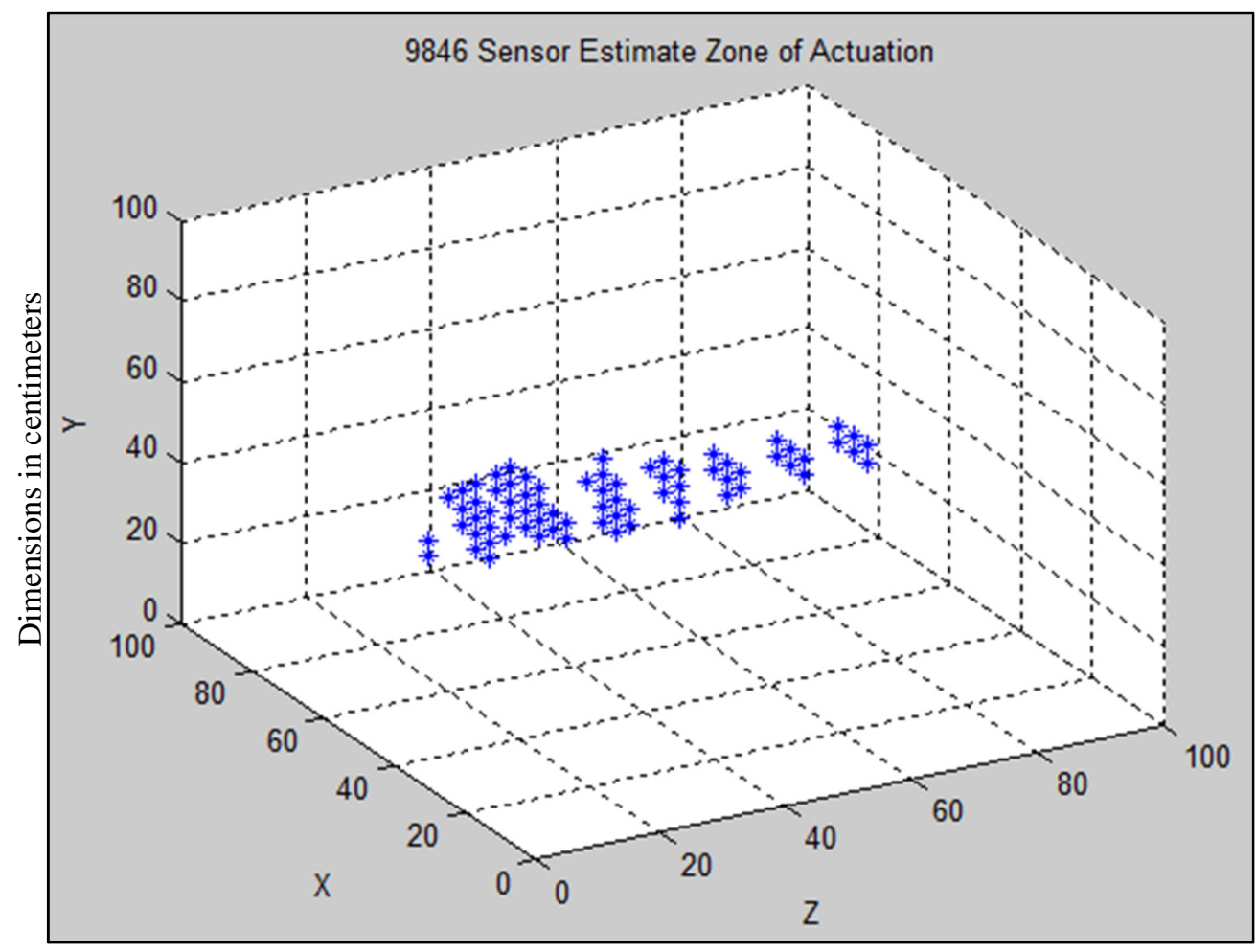

Figure 6. 9846 LEGO's ultrasonic sensor estimate zone of actuation

The planes mapped suggest the response threshold of the sensor. In the eleventh plane, the sensor could not detect the presence of the plate in any of the grid points $\mathrm{X}, \mathrm{Y}$.

It is possible to see that the sensor installed in the center of the first plane, coordinates $[\mathrm{X}(-500 \mathrm{~mm}), \mathrm{Y}(500 \mathrm{~mm})$, $\mathrm{Z}(0.0 \mathrm{~mm})]$, emits acoustic waves able to detect a plate $\left(40 \mathrm{~mm}^{2}\right)$ in a space not exceeding $1000 \mathrm{~mm}$. The characteristics of the sensor, as reported in its specifications, do not transmit accurate information about the actuation zone.

It can be seen that, the Figure 6 suggests the actuation zone of the ultrasonic sensor 9846, where the behavior of the acoustic field cannot be analyzed only as a top view.

According to the result of the graph, the plane positioned at $100 \mathrm{~mm}$, in front of the sensor, the reading was able to detect the object in only two points. Then, for the next planes, the number of points rises. For the planes positioned after the $400 \mathrm{~mm}$ from the sensor, it was possible to see a reduction of the detection, i.e. the sensor start losing the capability to detect the plate in function of the distance. With the results, five planes (matrices) of 625 points were joined, forming a matrix $3 \mathrm{D}$, which presents the estimate zone of actuation.

The results show that the best distance detection of sensor is between 200 and $400 \mathrm{~mm}$. After this distance, there is a reduction of the actuation zone. In the plane located $1000 \mathrm{~mm}$, the sensor has not detected points, which suggests be the maximum distance range of the sensor, when using a $40 \mathrm{~mm}$ square plate.

For better comprehension, the figure 7 presents the three views of the actuation zone obtained by the mapping, where is possible to distinguish the difference between the horizontal section (commonly used in datasheets) and the 3D actuation zone.
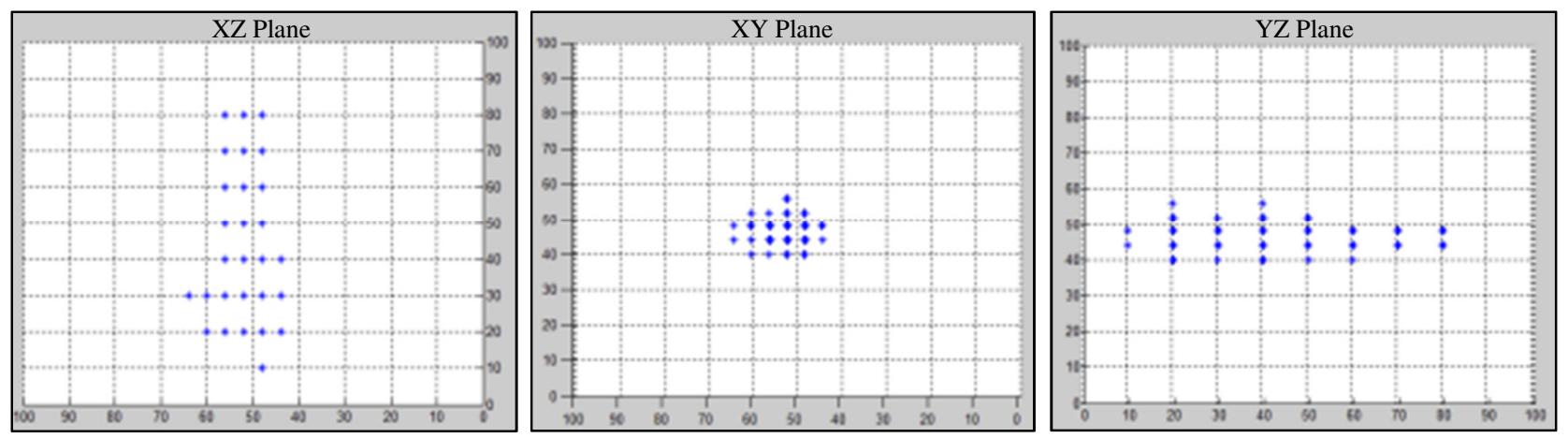

Figure 7. Top, front and side view of the estimate zone of actuation of the 9846 ultrasonic sensor 


\subsection{Second experiment}

According to the specifications of Elec Freaks, the ultrasonic sensor, model HC-SR04, is capable of measuring the distance of objects between 20 and $4000 \mathrm{~mm}$. The scan was performed in a space of $2000 \mathrm{~mm}^{3}$, using the steel plate of $40 \mathrm{~mm}^{2}$. For the $\mathrm{Z}$ axis of the structure, in this experiment, it was segmented into several planes, considering a distance of $100 \mathrm{~mm}$ from one to another. Thus, twenty planes, parallels of the first plane, where the sensor was positioned, were analyzed. In each one of these, the plate was moved, creating a mesh of points in $\mathrm{X}$ and $\mathrm{Y}$ axes, forming through the detection points, the estimate zone of actuation of the sensor, as shown in Figure 8.

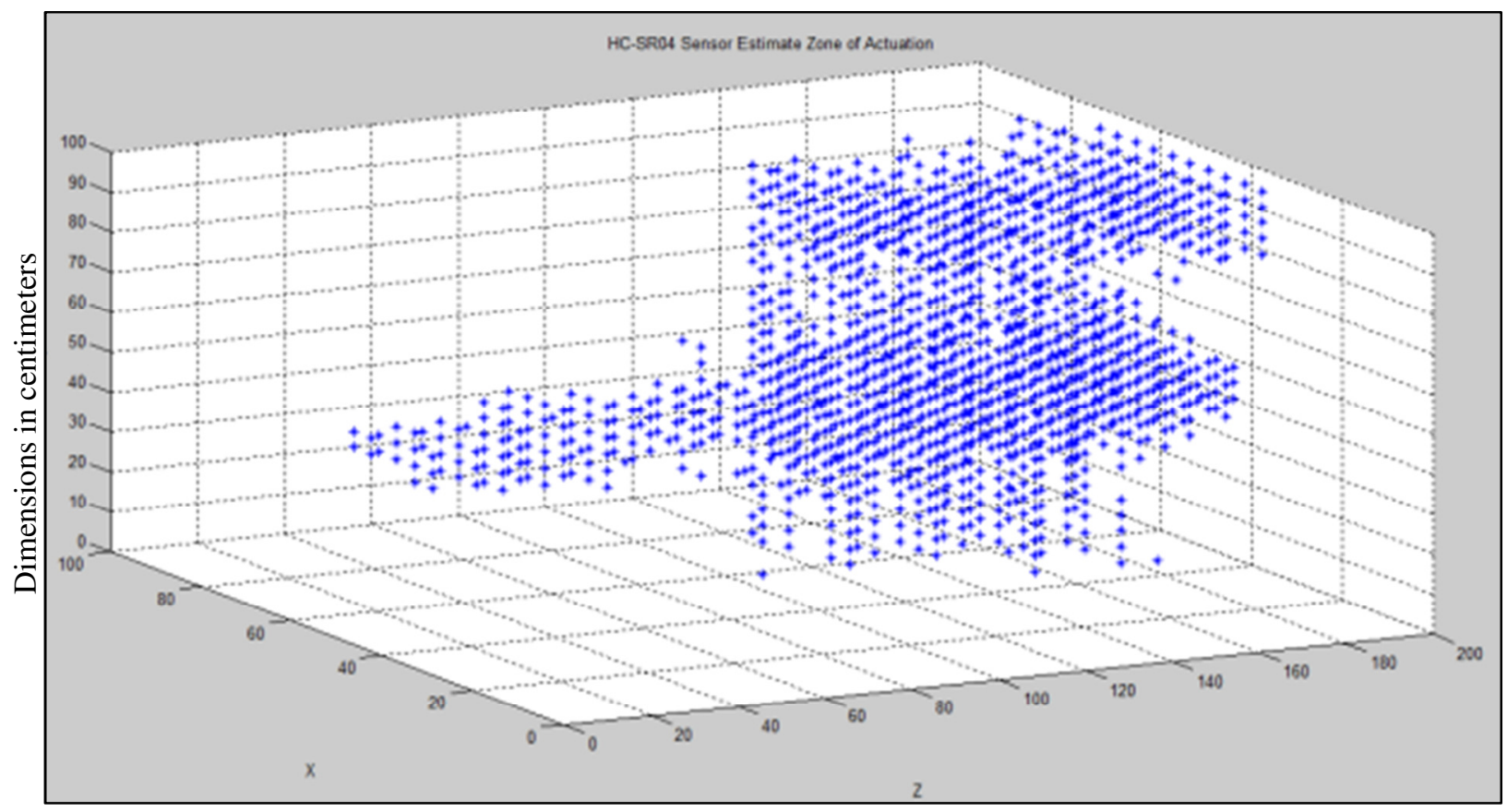

Figure 8. HC-SR04 ultrasonic sensor estimate zone of actuation

The twenty mapped planes suggest the response threshold of the sensor. In the twenty-first plane, the sensor could not detect the presence of the plane in any of the grid points $\mathrm{X}, \mathrm{Y}$.

It is possible to see that the HC-SR04 sensor is more robust than the sensor 9846. It is commonly used in more complex projects, when compared to the 9846 sensor. However, distinct of what is mentioned in the technical specifications, the capability to detect an object, does not exceed $2000 \mathrm{~mm}$, when is used a plate of $40 \mathrm{~mm}^{2}$.

The characteristics of the sensor, detailed in the product datasheet are not sufficient to define the actuation zone. Figure 7 meets this need, suggesting an estimate zone of actuation for the HC-SR04 ultrasonic sensor. According to the results of this experiment, was possible to understand that the angle of actuation (15 degrees), mentioned in the datasheet, occurs vertically. This information was not clear before the experiment.

The results show that the best distance detection sensor is between 1000 and $1800 \mathrm{~mm}$. After this distance, there is a reduction of the actuation zone. Furthermore, comparing the estimate actuation zone with the result of horizontal mapping (top view), it was revealed that the behavior of the acoustic field cannot be analyzed only as an horizontal section. In many points, upper and lower, in relation of the sensor, the plate could be detected, confirming the benefit of three-dimensional analysis of the actuation zone.

For better comprehension, the figure 9 presents the three views of the actuation zone obtained by the mapping, where is possible to distinguish the difference between the horizontal section (commonly used in datasheets) and the 3D actuation zone.

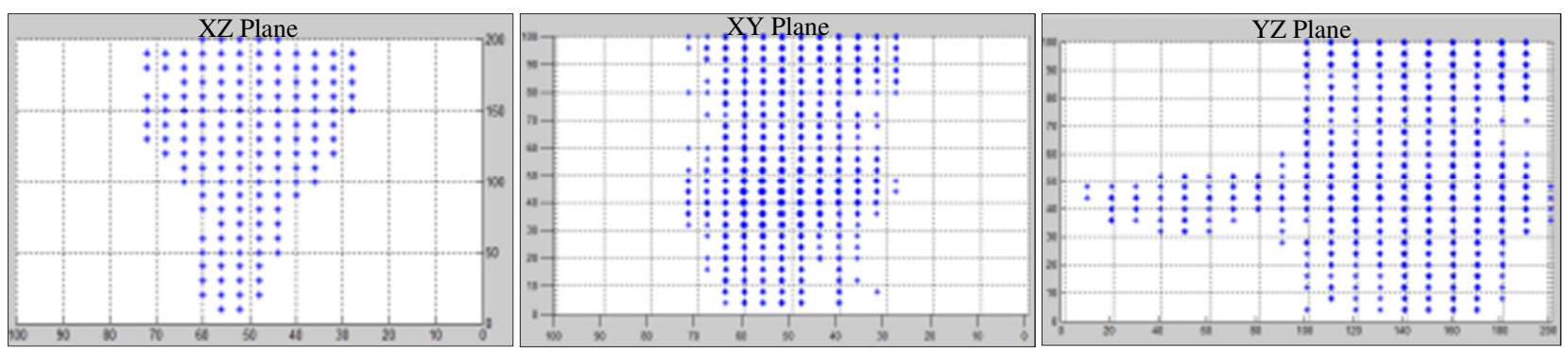

Figure 9. Top, front and side view of the estimate zone of actuation of the HC-SR04 ultrasonic sensor 


\section{CONCLUSION}

The results obtained with the mapping of ultrasonic sensors indicate a better definition of the actuation zone, allowing a better interpretation of the operating space. This result can be included in the datasheets of the sensors as additional information of high importance, which contributes for applications that requires this kind of information.

Based on variations of industrialization, voltage, among others, it is not possible to say that all models of the sensors analyzed, have the same behavior when compared to those who were not testing.

The use of this method of mapping has proven efficient, ensuring better interpretation of the actuation zone, which contributes for applications that requires a defined space. In cases such as proximity detection between vehicles, that demands high levels of safety and reliability, the method would facilitate in the identification of the kind of sensor to be applied and the best position of it.

For application of ultrasonic sensors in vehicles, aiming to detect approximation others vehicles in the actuation zone, the knowledge of this space is indispensable, especially for the correct positioning of the sensor. With a predefined space, would be possible to plot the actuation zone in computer systems engineering, simulating the best positioning of the sensor in the vehicle.

For future experiments, test will be applied using objects with different dimensions, evaluating if in function of the sensors frequency, the results can change. The plate of $40 \mathrm{~mm}^{2}$, was randomly chosen, once that biggest objects would cover more space in the structure, so could influence in the determination of the actuation zone. Furthermore, experiments with objects with different forms and in others planes (not perpendicular to the sensor), will contribute to better definition of the actuation zone.

\section{ACKNOWLEDGEMENTS}

The authors gratefully acknowledge the financial support by CAPES, FEMEC/UFU, FAPEMIG, PPGGO/UFG/RC, FAPEG and CNPq.

\section{REFERENCES}

Bastos, T., Abreu, J., Ceres, R. 1992. Uso de Sensores Ultra-Sônicos na Medição de Parâmetros em Robótica e Outras Aplicações.

Borenstein, J., Koren, Y. 1988. Obstacle Avoidance with Ultrasonic Sensors. IEEE Journal of Robotics and Automation. Vol. 4.

Fuentes, Rodrigo C. Apostila de Automação Industrial. Santa Maria: Universidade Federal de Santa Maria, 2005.

Kim, S. and Kim H. 2010. Optimally Overlapped Ultrasonic Sensor Ring Design for Minimal Positional Uncertainty in Obstacle Detection. International Journal of Control, Automation, and System.

Mágori, V. 1994. Ultrasonic Sensors in Air. Corporate Research and Development, Siemens AG. Munich, Germany.

Majchrzak, J., Michalski, M., Wiczynski, G. 2009. Distance Estimation With a Long-Range Ultrasonic Sensor System. IEEE Sensors Journal, Vol. 9, No 7, july 2009

Mohammad, T., 2009. Using Ultrasonic and Infrared Sensors for Distance Measurement. World Academy of Science, Engineering and Technology.

Rastogi, R. and Mehra, R. 2012. Efficient Error Reduction in Ultrasonic Distance Measurement Using Temperature Compensation.

Thomazini, D., Albuquerque, P. 2005. Sensores Industriais Fundamentos e Aplicações.

Webster, J. 1999. Measurement, Instrumentation and Sensors. Handbook.

Zang, X., Mao, Y., Zhao, H., Guan, X. 2004. The Research of Ultrasonic Detection Method for Car Back Obstruction. Jilin University, CHangChun, China.

\section{RESPONSIBILITY NOTICE}

"The authors are the only responsible for the printed material included in this paper". 